
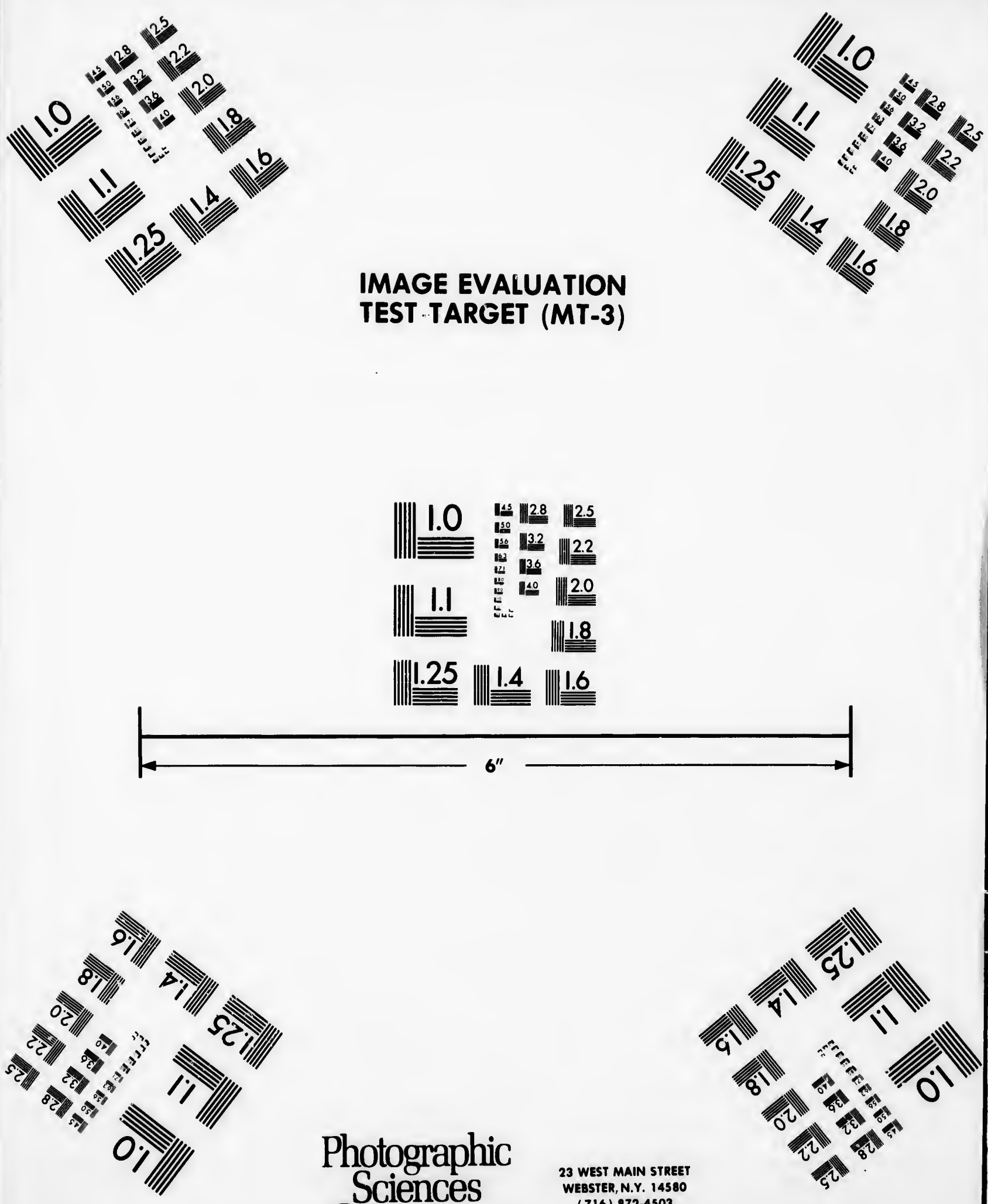

Photographic Sciences
Corporation

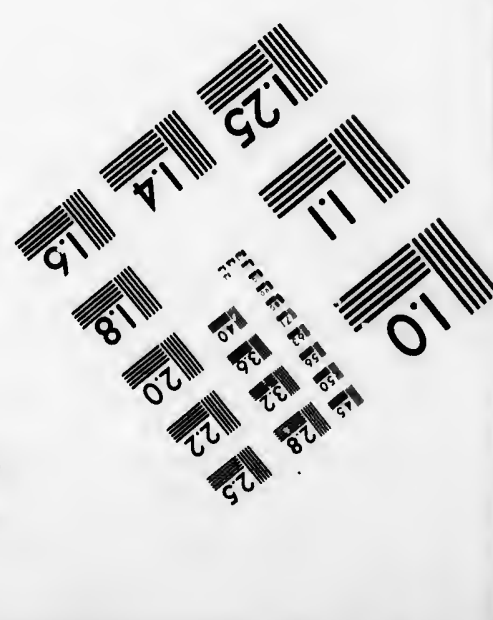




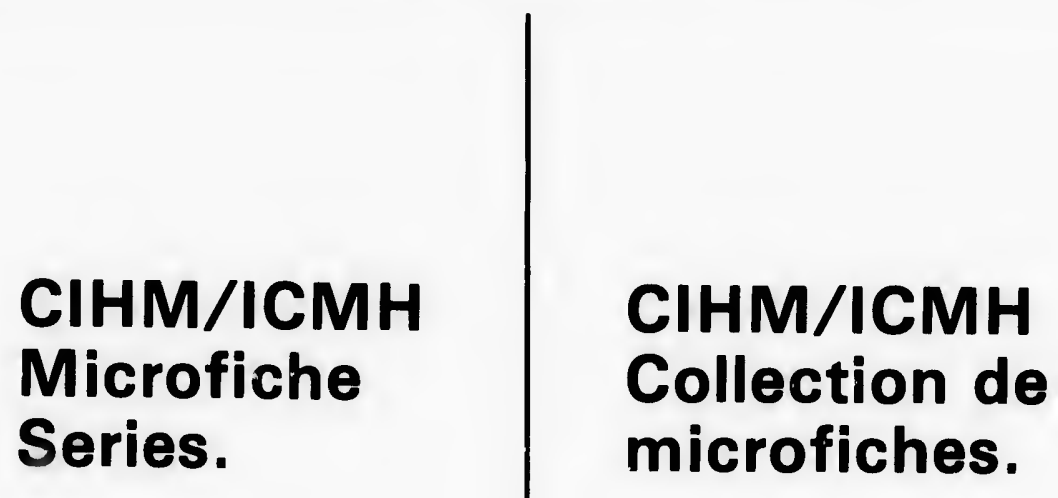

Canadian Institute for Historical Microreproductions / Institut canadien de microreproductions historiques
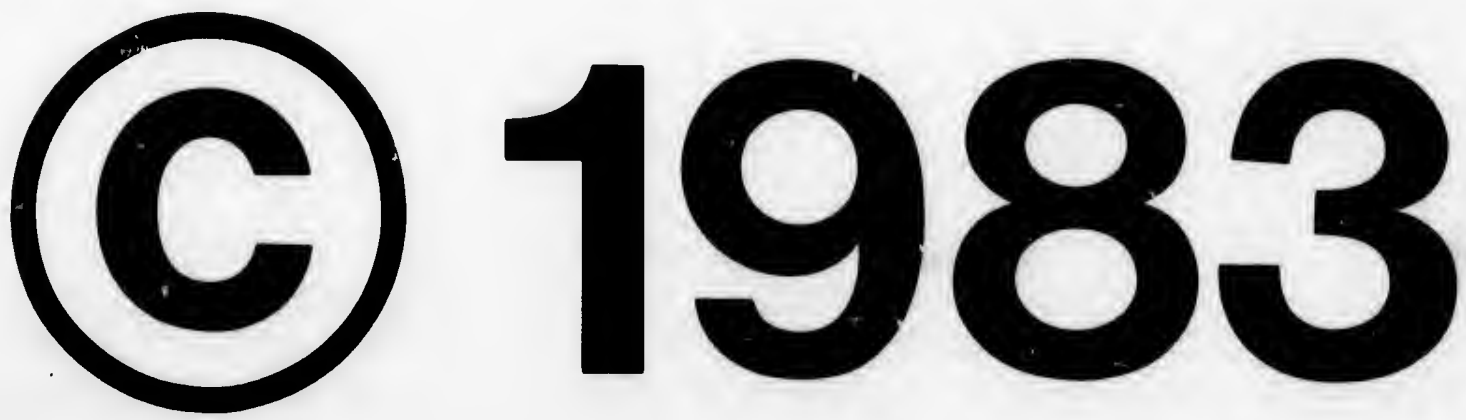
The Institute has attempted to obtain the best original copy available for filming. Features of this copy which may be bibliographically unique,

which may alter any of the images in the reproduction, or which may significantly change the usual method of filming, are checked below.

C sloured covers/

Couverture de couleur

Covers damaged/

Couverture endommagée

Covers restored and/or laminated/

Couverture resteurée et/ou pelliculée

Cover title missing/

Le titre de couverture manque

Coloured maps/

Cartes géographiques en couleur

Coloured ink (i.e. other than blue or black)/

Encre de couleur (i.e. eutre que bleue ou noire)

Coloured plates and/or illustrations/

Planches et/ou illustrations en couleur

Bound with other material/

Relié avec d'autres documents

Tight binding mey cause shadows or distortion along interior margin/

La re liure serrée peut causer de l'ombre ou de la distortion le long de la marge istérieure

Blank leaves added during restoration may appear within the text. Whenever possible, these have been omitted from filming/

II se peut que certaines pages blanches ajoutées lors d'une restauration apparaissent dans le texte, mais, lorsque ceia était possible, ces pages n'ont pas été filmées.

\section{Additional comments:/}

Commentaires supplémentaires:
L'Institut a microfilmé le meilleur exemplaire qu'il lui a été possible de se procurer. Les détails de cet exemplaire qui sont peut-être uniques du point de vue bibliographique, qui peuvent modifier une image reproduite, ou qui peuvent exiger une modification dans la méthode normale de filmage sont indiqués ci-dessous.

Coloured pages/

Pages de couleur

Pages damaged/

Pages endommagóes

Pages restored and/or laminated/

Pages restaurées et/ou pelliculées

Pages discoloured, stained or foxed/

Pages décolorées, tachetées ou piquées

Pages detached/

Pages détachées

Showthrough/

Transparence

Quality of print varies/

Qualité inégale de l'impression

Includes supplementary material/

Comprend du metériel supplémentaire

Only edition available/

Seule édition disponible

Pages wholly or partially obscured by errata slips, tissues, etc.. have been refilmed to ensure the best possible image/ Les pages totalement ou partiellement obscurcies par un feuillet d'errate, une pelure, etc., ont été filmées à nouveau de façon à obtenir la meilleure image possible.
The co to the

The in possib: of the filmin

Origin begin the le: sion, other first p sion, or illu:

The lo shall TINUE which

Maps, diffore entirel beginr right requir metho

This item is filmed at the reduction retio checked below/ Ce document est filmé au taux de réduction indiqué ci-drssous.

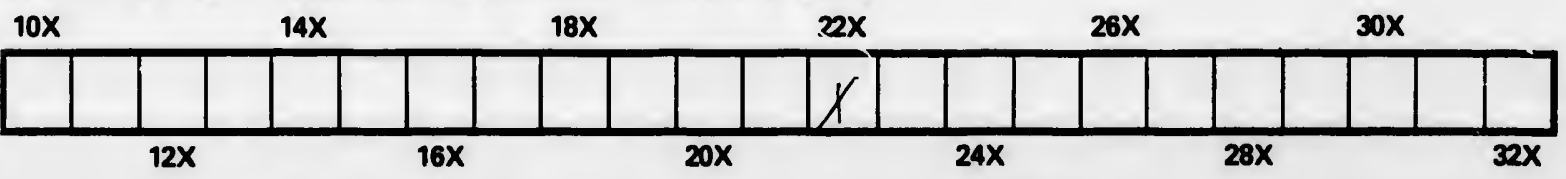


The copy filmed here has been reproduced thanks. to the generosity of:

New Brunswick Museum

Saint John

The images appearing here are the best quality posaible considering the condition and legibility of the original copy and in keeping with the filming contract spocifications.

Original copies in printed paper covers are filmed beginning with the front cover and ending on the last page with a printed or illustrated impression, or the back cover when appropriate. All other original copies are filmed beginning on the first page with a printed or illustrated impression, and ending on the last page with a printed or illustrated impression.

The last recorded frame on each microfiche shall contain the symbol $\longrightarrow$ Imeaning "CONTINUED"), or the symbol $\nabla$ (meaning "END"). whichover applies.

Maps, plates, charts, stc., may be filmed at different reduction ratios. Those too large to be entirely included in one exposure are filmed beginning in the upper left hand corner, left to right and top to bottom, as many frames as required. The following diagrams illustrate the method:
L'exemplaire filmó fut reproduit grâce à le gónórosité de:

New Brunswick Museum

Soint John

Les images suivantes ont dté reproduites avec le plus grand soin, compte tenu de la condition et de la netteté de l'exemplaire filmb, et an conformité avec les conditions du contrat de filmage.

Les exemplaires originaux dont la couverture en papier est imprimbe sont filmés en commençant par le premier plat et en terminant soit par la dernibre page qui comporte une empreinte d'impression ou d'illustration, soit par le second plat, selon le cas. Tous les autres exemplaires originaux sont filmbs en commençant par la premidre page qui comporte une empreinte d'impression ou d'illustration et en terminent par la derniere page qui comporte une telle empreinte.

Un des symboles suivants apparaitra sur la dernidre image de chaque microfiche, selon le cas: le symbole $\rightarrow$ signifie "A SUIVRE", le symbole $\nabla$ signifie "FIN".

Les cartes, planches, tableaux, etc.. peuvent ètre filmés à des taux de réduction différents. Lorsque le document est trop grand pour être reproduit en un seul cliché, il est filmé à partir de l'angle supórieur gauche, de gauche à droite, et de haut en bas, en prenant le nombre d'images nécessaire. Les diagrammes suivants illustrent la mothode.

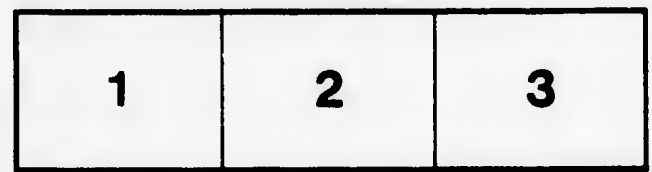

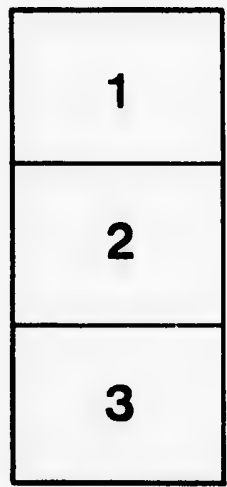

\begin{tabular}{|l|l|l|}
\hline 1 & 2 & 3 \\
\hline 4 & 5 & 6 \\
\hline
\end{tabular}




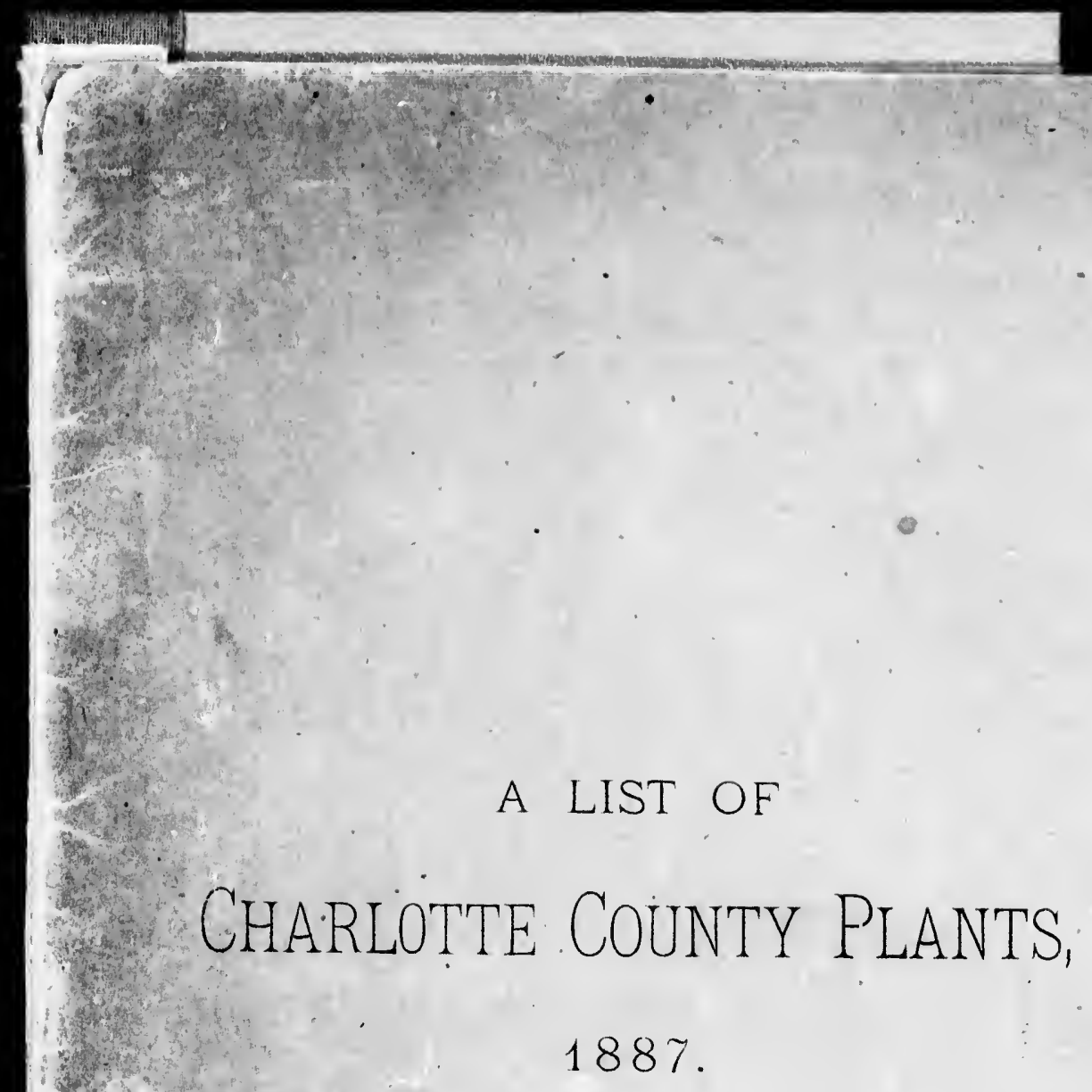




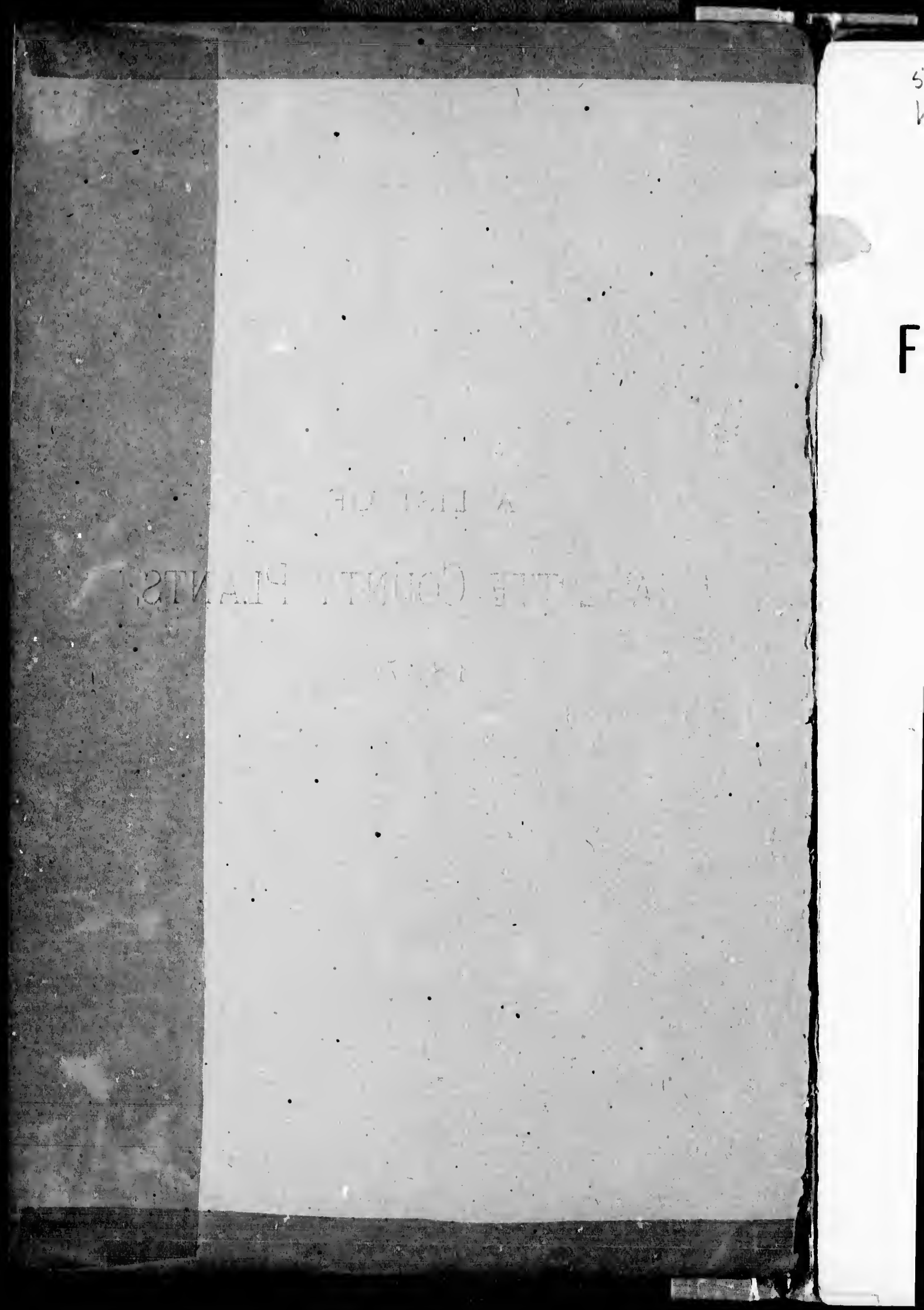




\subsection{3 \\ 1984}

A LIST OF

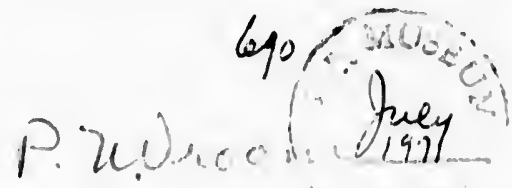
Plat
piatucter Lie!.

\section{Flowering Plants and Ferns} Furin IX

\section{Charlotte County, New Brunswick}

IS J. VROON

And other Members of the Botanical Committec of the Natmal History Society of New Brunswete.

ST. STFPIIFN, N. B.:

COURIER STEAM PRINTING IIOUSE 


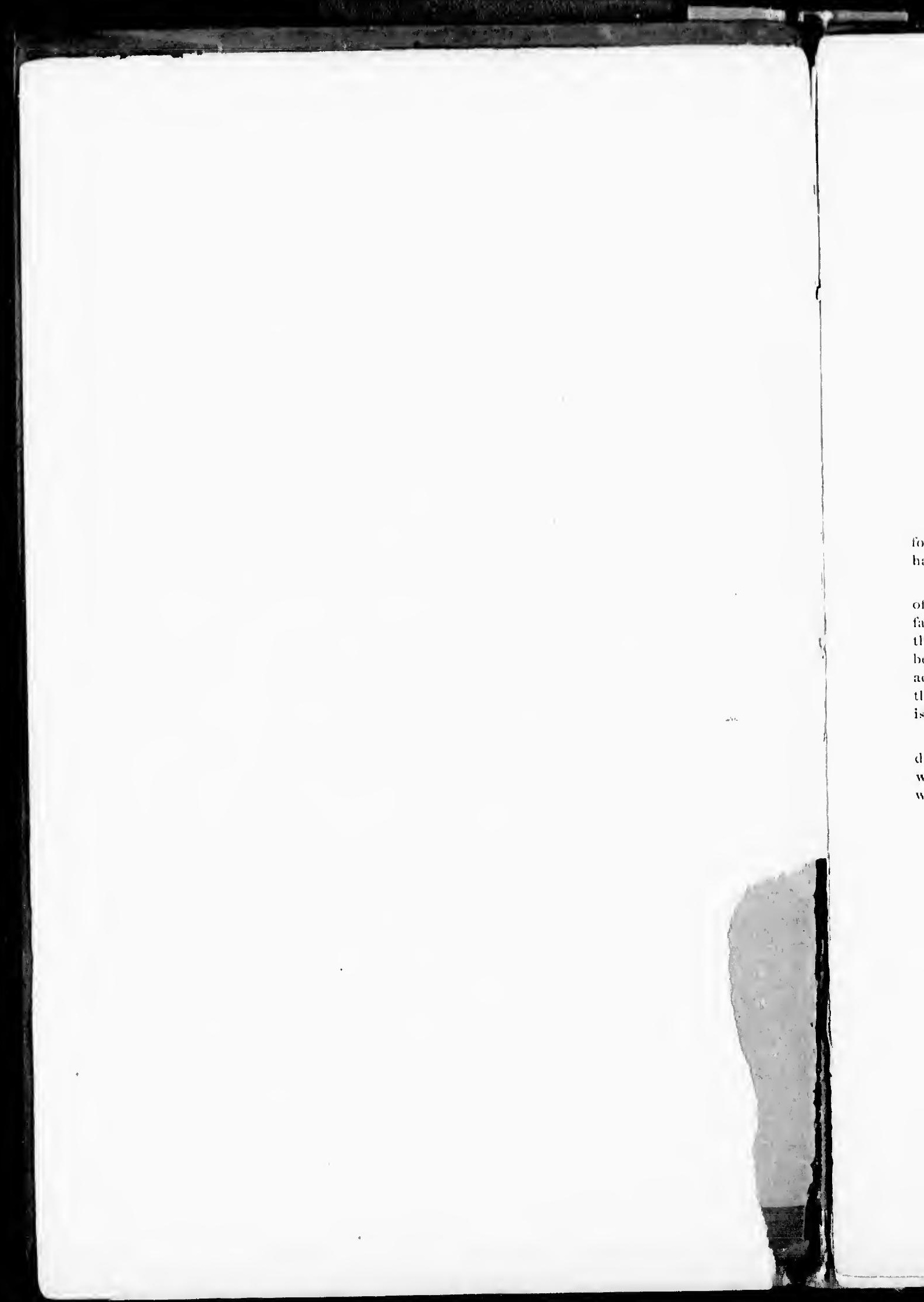


NoTE.

This List is thought to contain the nancen of most of the Fowering Plants and Ferms found in Charlofte Comb up to the present time. with the exception of Sedges, which have not been carefully stidied, and a few of the l'ondwedels and Anters.

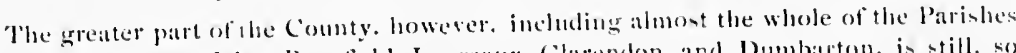
of Sant Crois, West Inles, Penntiedd. Lepreatux. (Clatendon, and Dumbaton, is still, sor far as the writer is aware, unvisited by the plant collector: and excepting firge's landand, the site of a summer camp of the Natumal Ilintory Societs, the only sections that have been well examired are in the neighborhool of Sant Andrews and Saint Stephen. The accompanying notes of dintribution. therefore, apply chictly to the southwentern part of the maindand. The probability of having hut few opportunities for field work in the future is the only excuse for presenting the list in such an imperfect statc.

Prof. Macoun and Prof. Fowler have kindly determined diflicult species. Thanks are due to several collectors for notes and specimens; especially to Mr. IV. F. Ganong, by whose assistance the lint has been much extended. For the defiects of proof reading, the writer alone is responsible

St, Stephen, Narch, is 85 . 


\section{LIST OF CHARI.OTTE COUN'TY PLANTS.}

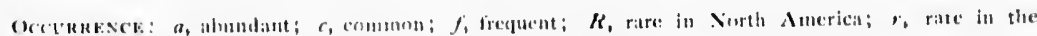

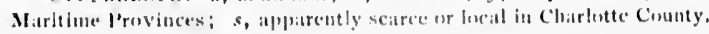

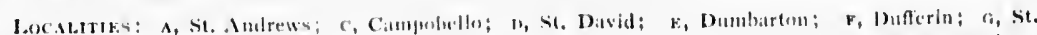

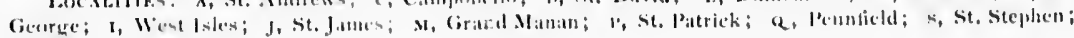
x, st. Cruix.

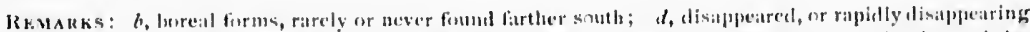

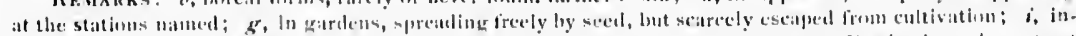

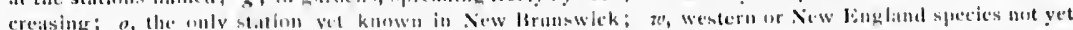

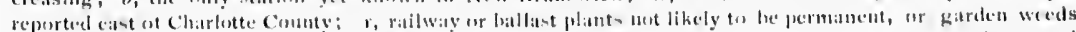

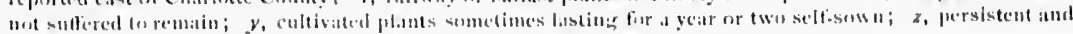

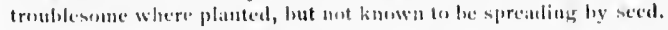

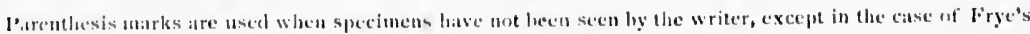

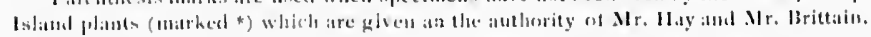

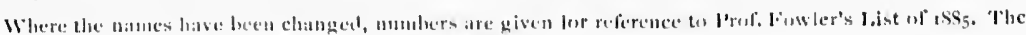
marho of uncertianty ajply to the determination uf species.

\section{PIANEROGAMIA.}

ANGIOSPERME.

Clemati

RINUNCOLACEA.

verticillaris IDC $A$ s

- Virginiana L $c$

Thalictrum

- polygamum Muhl $c[t]$

Ancinome

diche toma I, *

nemorosia $\mathrm{L}$ s

- llepatica L $r$ sE

Rantunculus

aquatilis var trichophyllus

$$
\text { Gr } r \text { s }
$$

Flammula vatr replans Ney Combalaria Pursh s.l(G) abortivus $L$ sceleratus L $r$ s

$=$ acris $L$ refens $L$ hispidus Mx

Coptis

= trifolia Salish $a$

Aquilegia volgaris $L$ is

Actie:

albu Bigel $s$

spicata var rubra $A$ it $f$
BERBERIDACE.E

\section{Berberis}

zulgaris lo $z$

NYMIILACLA:

[Brasenia

- peltitia Pursh f

Niuphar

advena $\mathrm{Ait} c$

Nimplacia

- odoritit Ait $f$

_. var minor sims J

SARRACENLACEA.

Sarraceniat

purpurea I.

\section{PAPAYERACEA}

\section{lapaver}

sommiferum Lo $g$

= lubium $\mathrm{L} g$

FITATRIACL.T.

Dicentra

Cucullaria DC $s$ c

Artlumiar cirrhosa Ruf sr

Corvalalis crlatuca Pursh $f$

Fillmariat officinalis $\mathrm{T}$ si
CRECHERR:

Nisturtium ollicinale $\mathrm{RBr} r$ palustre 1)C Armoracia Fries $z$

Cilvolimatne prittensis l. K r od hirstuta L,

Dentari:

- diphylla Mx s

Sisvimbrium

officinale Scop, $\mathrm{s} i$

Brassica

Sinufistrum Boiss

nigra Koch

Ile'speris

matronalis $L, g$

Capsiclla

Bursa-Pusturis Manch a

Calile

Americana Nutt $c$

VIOIACE.T.

Violit

- nalmata var cucullata Gra

sarittata $A$ it $G o$ ?

odorata $L, g$

blanda Willd $c$

- var palustriformis $\mathrm{Gr}$ ? - var renifolia $\mathrm{Gr}$ ? [66] 


\section{primulifolla I. Ao \\ panceulista I. s (s) Ne; \\ pulesecell dit sis d \\ canina var Muhlentergii

$$
\text { (ir } f[(x)]
$$ \\ - tricolor l. 1}

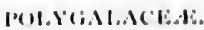

Polyegilat

pancitolia Willd $r$ s

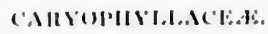

\section{Sip punar)rit}

uflicinulis H. H. $\mathrm{fl.} z$

verecriria lo si al

Siling'

intiate Sint

Armericl l, y

"octifloral.

\section{le 'cllillis}

Githago Latm

zesfertiun Sibth s out

Cirastium

viscosum l, $c$

arieuse l, s .

Stellarial

modice $S_{m}$ " longifolia N/ubl ?

longipes Goldie s

boreitlis Bigel, is

lamifisasa Rottb * rb

Aremaria

litterifloria I, c

Silgriona

procumbens I, $c$

(nodosa E Meyer $r$ si)

Sperymlat

arvensis $L$ c

spergularia

salina l'resl

media Presl

\section{Pi)rtulaca \\ PORTUACACE.S. \\ oleraceal, \\ Clantomiat \\ - Caroliniana Mx s sc;}

IYPIERCACI:AL.

Hypericum ellipticum IIook

- ferforatum $t$. mulilum L Camadeune I.

Elodea 'iuupanulata l'ursls

$$
\text { Maláa MALVACE.E. }
$$

rotundifolia $l, r \wedge d$ moschital.

IIibiscus

Trionum l. $\mathrm{s}$

\section{1,101,1:}

'l'iliil

- Americuna I. s

LINACER:

LinIIII

"situtisuimuml.

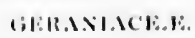

Geraniun

- Cimfolinianum if $f$ Roberlianumu 1, ;

()xilis

- Sieterillat I,

(o)ruiculata var stricta Say

Imprations

- tilloir Nill.

llex

II.KINISI:

- verticillalla Gir s lis

Nemopanthes

C'ammlensis I) $f$

MIAMNACLA.

Rhammus

alsifolia Liller s J

S.MISIMCI.l.

Acer

Pennsylvanicum I, spicatium Litul

sisceluatrinum Wang rubrum $\mathrm{I}$,

\section{A.ACORDIACI.J.}

Rhus

typhina I. "

= Foxicodendion I. $f$

\section{IEGTINos.ti.}

\section{Maticagro}

lufulinal.

Melilotus:

alba latum

officinulis Willd

Tirifolium

arvense l., $\mathrm{b}(1)$

protensel.

repens l.

hlbridum l. fi

"igrarium $l$, $\mathrm{JS}(\mathrm{M})$

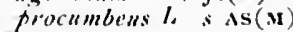

Robinier

viscosa lent z

Ornithopus:

scorpioides DC R $\mathrm{s} x$

Desmodium

Canadense i) $s \mathrm{~J}$

Vicia

saliz'a z'al angrastifolia Ser.

Cracea $\mathrm{L} s$
I.athirus

- maritimus Bigel

r.luser var myrtiloblius (ir

Aimpliciarpara

monoica Ell \&

Apios

tuberoma Maench s js

\section{IIISICl:A..}

Promuls

(pumilat l,, (b)

- Penmovilranica Ifi r

Virginianal 1

serotina Ehrh

Spiras

malicifolia I, c

- tomsentosal I, a

Ruluss

udoratus lo z

Chamemorus L cos(खण) /

Dalibariala $\mathrm{L}$

- tritlorus Rich

strigesus .IS

villowas viu frondosus lorr

- Cinadensis I. hispidus I.

(jellim

althum Gumel rs

strictuII $\Delta$ it $s \wedge(1 i)$

rivale I, $f$

Frisuaria

Virginiana Ducluesse

vene'a $I$. s

Putentillis

Norvegien I.

argenteal $1 . r$ sta

(arguta l'ursh $r s$ )

palustris scop e

firuticona L $r \mathrm{Jci}(\mathrm{N})$

triclentata sol $f$

Alsterisa 1 .

- Camadinsis var simplex T. Cir $c$

Agrimonial

Eupaloria I.

Rus:1

cimuamonn'al. $z$ ?

imicrantha $S$ m $z$

Carolinat I,

- lucida Fhro [193]

niticla Willd $s \mathbf{J}(\mathbf{G})$

(humilis Marsir $x$ )?

I'iins

arbutifolia var melanoe:arpallook $c$

Amerieana DC $f$

Cratiegus

- tomentosa I

Amalanchier

- Canadensis T\&Gr " - var oblongifolin $T \& G r$ - var oligrocarpa $\mathrm{Gr}$
1

II

$\mathrm{Cl}$

Ri

Sic

P.

I)

II

$\mathrm{P}^{2}$

II

I

E

I

c 


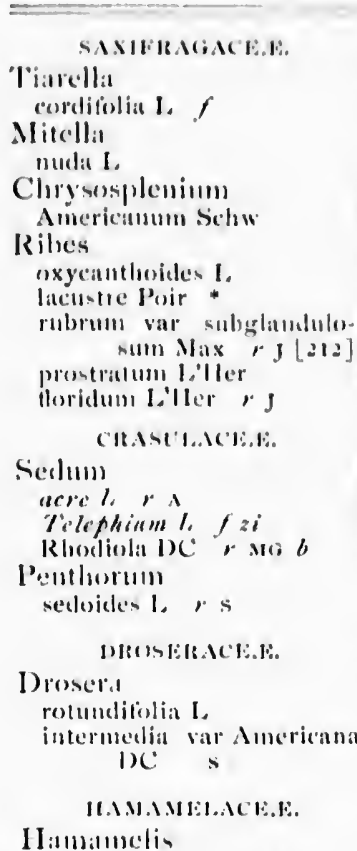

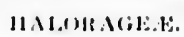

Proserpinatial palustris $\mathrm{L} R \boldsymbol{l}$

IIipinarls vuluaris 8 I

Mirioplinllam tenelluin Bigel $r \mathrm{o}$

Callitriche verua $\mathrm{J}, \mathrm{c}$

ONAGIRACH.S.

Epilohium spicalum Iam palustre var lineare $\mathrm{Gr}$ coloratum Nuhl ?

Lushigial palustris Ell

(Einothera hienuis L, [Virieties undetermined.] pumila L

Circas alpina $I$,

UMBELITERI E.

Iydrocotyle

Americaua $\mathrm{I}$,

Samicula Marilandica L J

\section{Cicutil}

maculatn 1. hollhitera I,

Cirr"l")

- Carruil. s

silum cicutitfollum Ginel $c$

Osmorrlais: brevintis DC:

l.igusticinu Sicolicum I, r. (i)

'Thisplintm aurcum Xiıtt $r$ * ficlimum Carmelemse Mx $r *$

Cinloplentrims (imelini lectels $r *$ [250]

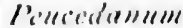
satioum $B$ di $/ / k$

Helitelenin

- hanatum . Mx

burucus:

- Carotar lo is

Cerecalis modesce lluds s $x$

\section{IIR.MIIACH.1\%.}

Arilia

racemosa I, s I

bispiclat Vent

nudicaulis I.

(trifilia Dec \& Pl , P)

(o) S.ACE.1\%

Cimmus

Cinnadeusis I. a circinatis L'Jfer :

-riceit I, s J

sentonitera Mix a alternitolia If $s . .(G)$

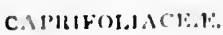

Simbucus

racemosa 1.

Canadeusis I.

Viburumm

- Lantanoides Mx c

- Opulus I. acerifolium I. \& $w$ dentitum I. Jis $u$ citssinoides $\mathrm{L}$, Lentago I y ito

Limlie'il

= borealis Gronos "

Lonicera cierulea I. $f$ ciliata Muli

Diervilla

- trifida Mrench

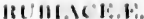

Ilomstomiat

cisuleu $\mathrm{l}$.

Cophalanthus

occiclentalis I, J $\pi^{4}$

Nitchellat

- repenal.

(jillium

ifuriune l, , *

tritidum var punillum (ir (- - var latilolium 'Tor (i) asprellum . Mx

tritiorum . Nx

\section{composite}

linpstoritum purpureum I. perfoli:tum $\mathrm{I}$.

Solidiarers

latifolia $\mathrm{l}, \mathrm{s}$

bicolor 1 .

(macrophylla l'ursh (i)

sempervitems I. s

puberulat Nutt $a$

speciosa Nutt $f$

rugosat Mill a

juncea dit $s$ ?

(serotinal dit 1")

Canalensis I, a

- nesuoralis dit a

lascecolata I. "

Aster

macrophyllus $J$.

radula dit

cordifolius I.

sagittifolius Willd ?

dittusus $\mathrm{Ait}$

salicifolius $\mathrm{Ait}$

Novi-Belgrii I, ?

pmicens I,

umbellitus Mill

acuminatus Mx

nemoralis $\Delta$ it $s(\mathrm{~N})$

I'ruhibly others: npecies en fuscel :utt uncertain.?

Erigeron

strigosus Mulal

Canadensis I.

Antennaria

plantaginifolia llook

Ainapualis

margaritacen Bsllk

Guaphalium

(polrceplat:m II,$x$ ) ? uliginosum I.

Inula

Ilelenium l. s s(rp)

Ambrosia

artemisiafolia $1, f$

Rudberkia

hirtal $l, f$ 


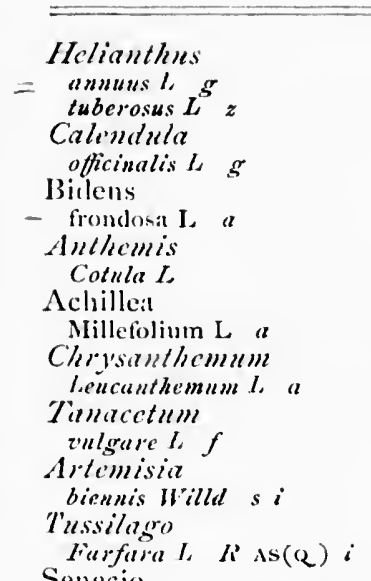

Senecio

aureus var Balsamita $T \& G$ vulgaris $L$ a

Erechtites

hieracifolia Raf $c$

Arctium

- Lafpa var minas Gr a

Cnicus

- areensis IIoffm a

lancrolatus lloffin a

muticus: Pursh

Cenlanra

nigra $L$ s $A$

Cyanks l,

Cichorium

Intybns $l$ s s

Tragopogon

pratensis $l R \mathrm{R} o$

Lcontodon

-autumualis $L$ c

IIjericium

Camadense Mx $r$ a

scabrum Mx $c$

Preninthes

serpentaria Pursh

alissima $\mathbf{L}$ ?

ficris:

echioides $I$ s $x$

T'araxacum

officinale Weber a

Litctuca

Canadensis L.

lencophisa $\mathrm{Gr}$ *

Sonchus

oleraccus $I$.

atser lill

ariensis $I$

\section{LOHELIACEAS}

Loheli:

- cardinalis $\mathrm{L} f \mathrm{~J} \operatorname{\mathrm {D}}(\mathrm{pE}) \mathrm{G} w$
Dortmanna L $f$ JUG( $(\mathrm{I})$ -intlata L $c$

CAMPANULACEAT.

\section{Campanulat}

rapunculojiles $L, r$ si $z$ rotundifolia $I$. \& MG aparinoiden Pursh $r$ gs

VACCINIACEAS.

Gaylussacia

dumosal TaGr $R \mathrm{~s}$ resinosa ToGr $f$

Vaccinitum

- Pennsylvanicum Lam a Canarlense Kalm corymbosum L $r$ s

- Vilja-Idiea L, a

Oxyeaceus palustris Pers macrocirpus Pursh c

Chiogenes

hispidula $\mathrm{T} \& \mathrm{Gr} \quad c$

Epigat

ERIC.ACLE:

- repens L $f$

Gaultheria

- proumbens I. $c$

Andromedar

polifolin $\mathrm{L} s$

Cissindra

calyculat:i Don $c$

Killuia

= angustifolia $\mathrm{L} a$ glauca $\lambda$ it $f$

Rhodedendion

- Rhodora Gmel a

Lediun

- latifolium Ait $\epsilon$

Chimathila umbellata Nutt

Moneses unitlora Gr $s$

Pyrola

secunda I.

elliptica Nutt rotundifolia $\mathrm{L}$.

\section{MTONOTROPE.E}

Monotropa

- unitlora l, c

I Iypopitys

lanutginosa Nutt $r A d$

PLAMAGINACEN.

Stitice

Limonium var Caroliı.j- Cuscuta ana $\mathrm{Gr} s$
PRIMULACEA.

'Trientalis

- Americana Pursh c

Steironema

ciliatum Raf

Ly simachia

strictil $\Lambda$ it $f$

thyrsitlora $\mathrm{L} \quad r \mathrm{~J}$

Glitux

maritima $\mathrm{L}$

Anagalis

ariensis l, r A

\section{OLEACLE.}

Fraximus

Americana I.

sambucitolia Lam

pubescens Lam $r \mathrm{~s}$

APCINACE.

Apocynum

androsatmifolium I

ASCLEPIANACEAS.

Asclepias

incarnata $\mathrm{L}$

GFNTINACEAS

Gentiana

linearis var latifolia $\mathrm{Gr}$ Je

II Ilenia

detlexil Griseb $s$

Mensintles

trifoliata $\mathrm{I}$.

Limnanthemum

latunosum Griseb $s$

(POLEMONIACEA)

(Polemonimm)

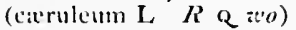

JORIRAGINACEAS.

Mertensia

maritima Don

borrago

officinalis $L$ g $\mathrm{s}$

Miosotis

laxa Ichm *

ariensis 11 oflim A

Symplaytum

officinale l. $\mathrm{s}(\mathrm{Q}) z$

CONVOINLIACH.

Convolvulus

-sepium var Americanus Sims $f$

Gronovii Willd s s(G)
So



$D a$

$S$

iis

$\operatorname{le}$

Lil

$\mathrm{C}$

$\overrightarrow{\mathrm{Ch}}$

$\mathrm{M}$

Ily:

I,

a

$\mathrm{Ve}$

A

s$$
\text { . }
$$

E

RI

$\mathrm{M}$

$\mathrm{E}_{1}$

Ut

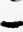

V

$N$

I. 


\begin{tabular}{|c|c|c|}
\hline 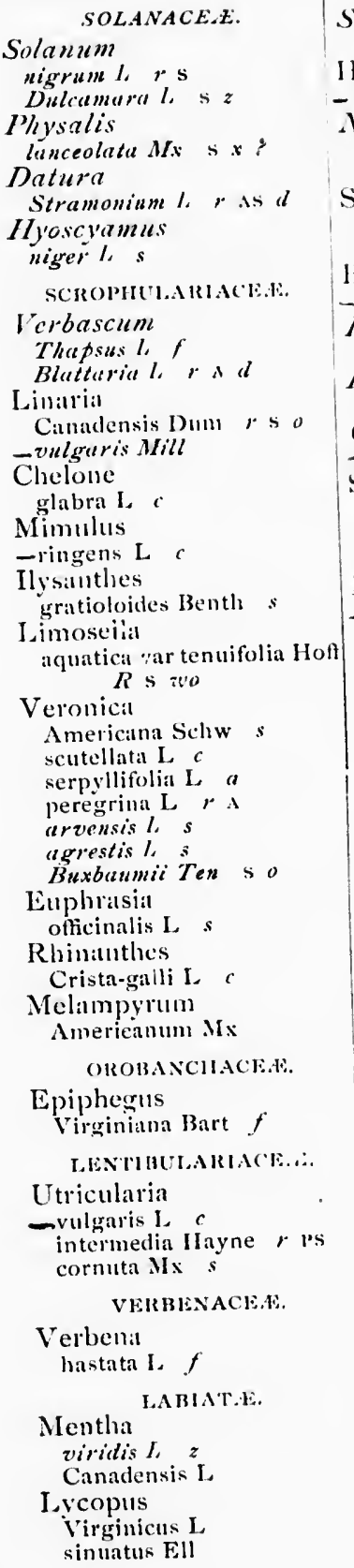 & 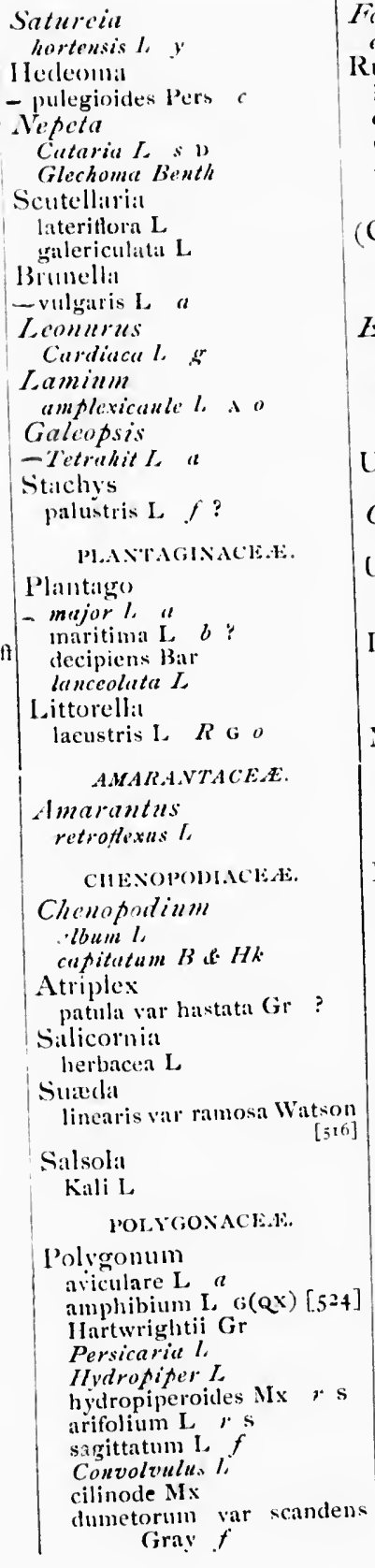 & 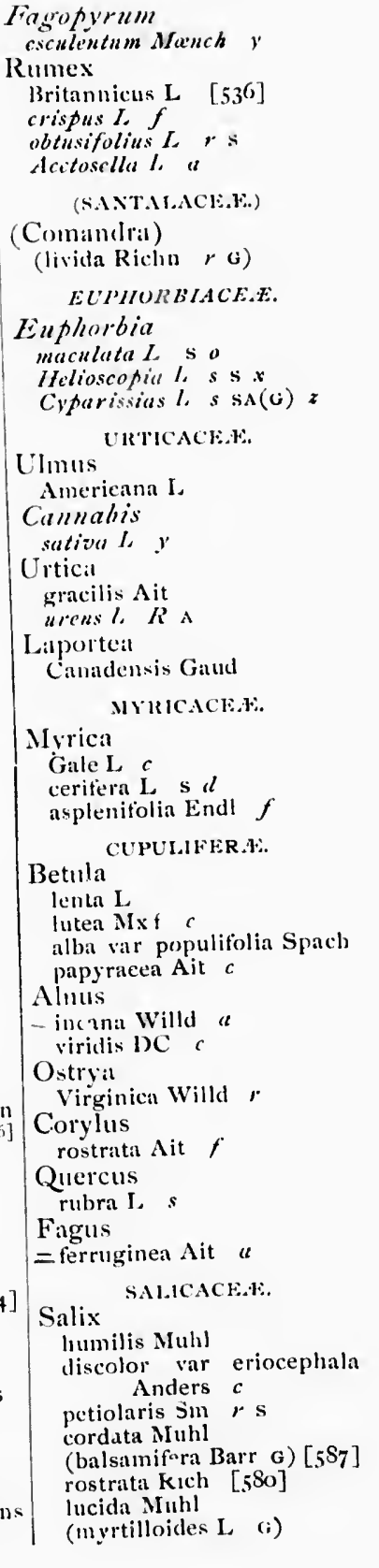 \\
\hline
\end{tabular}




\begin{tabular}{|c|c|c|}
\hline 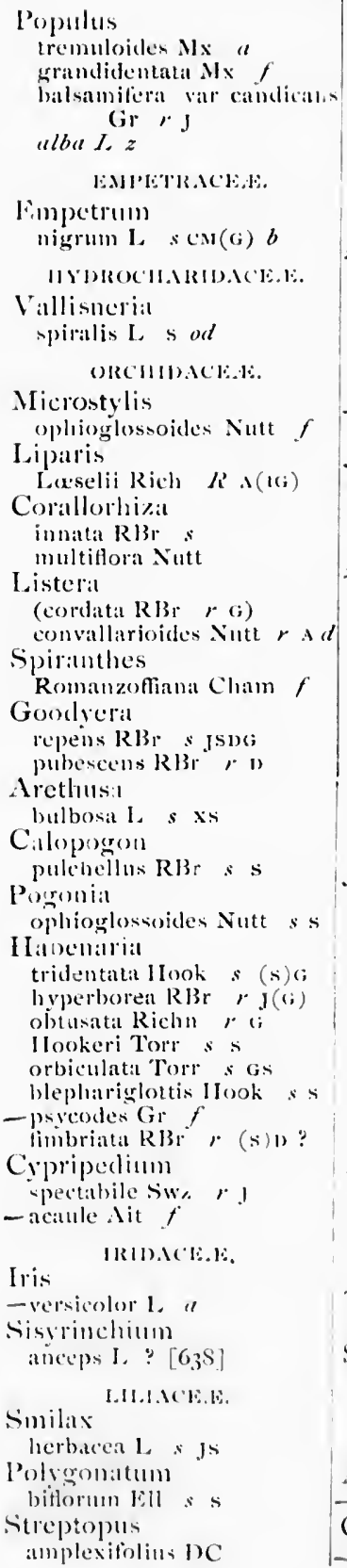 & 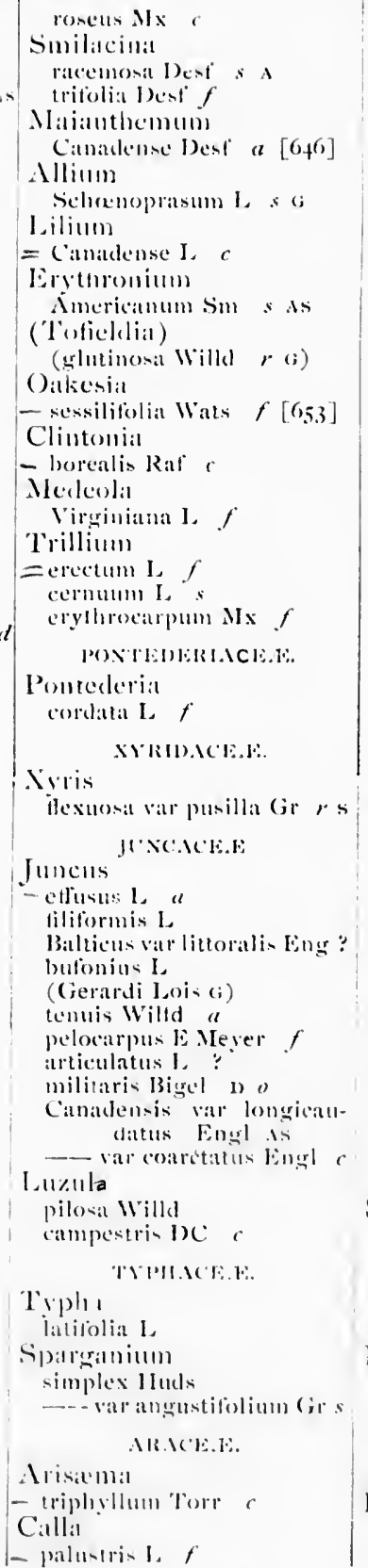 & 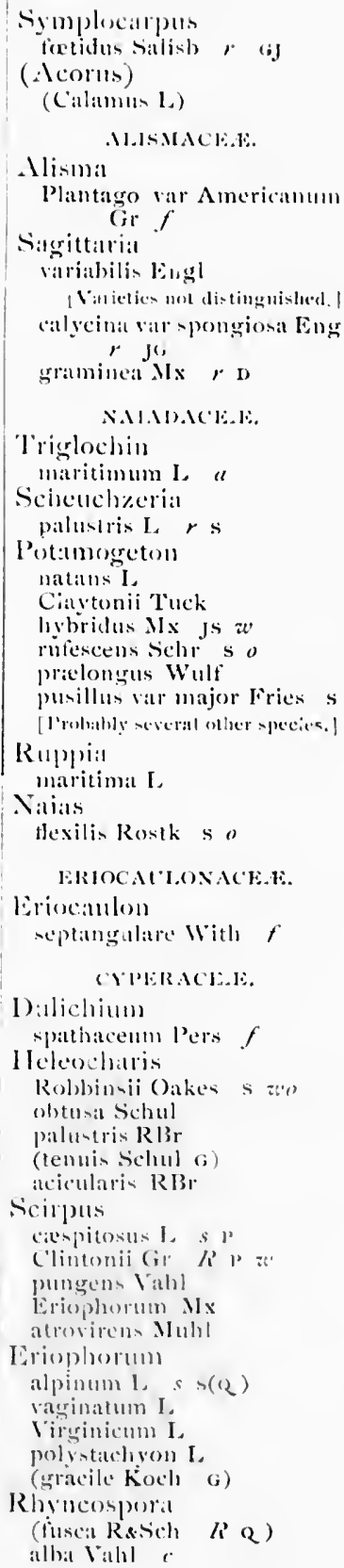 \\
\hline
\end{tabular}




\begin{tabular}{|c|c|c|}
\hline 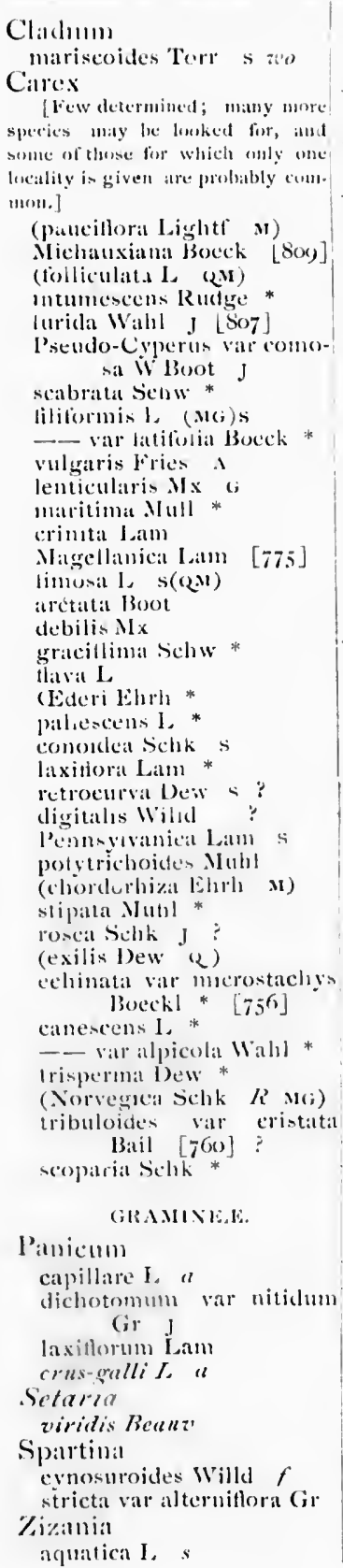 & 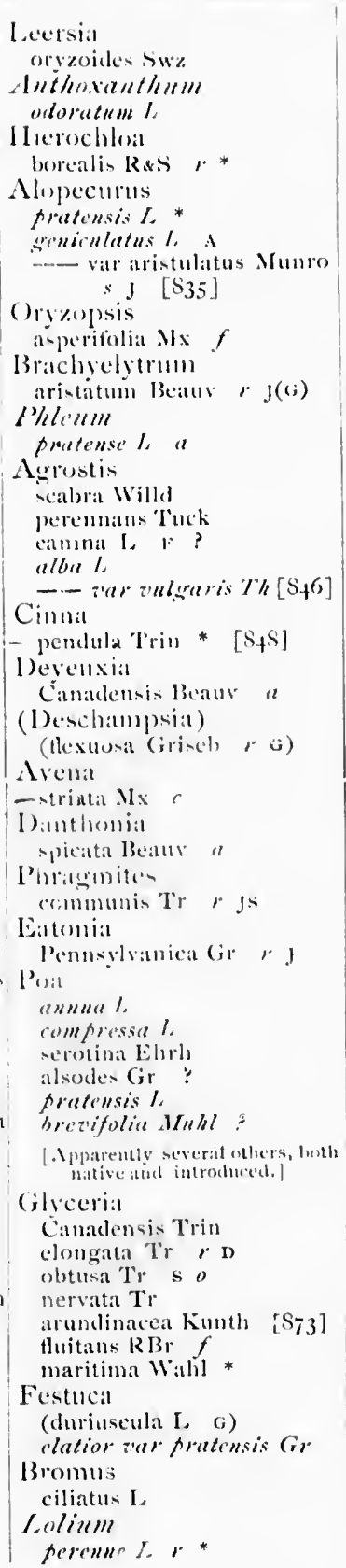 & 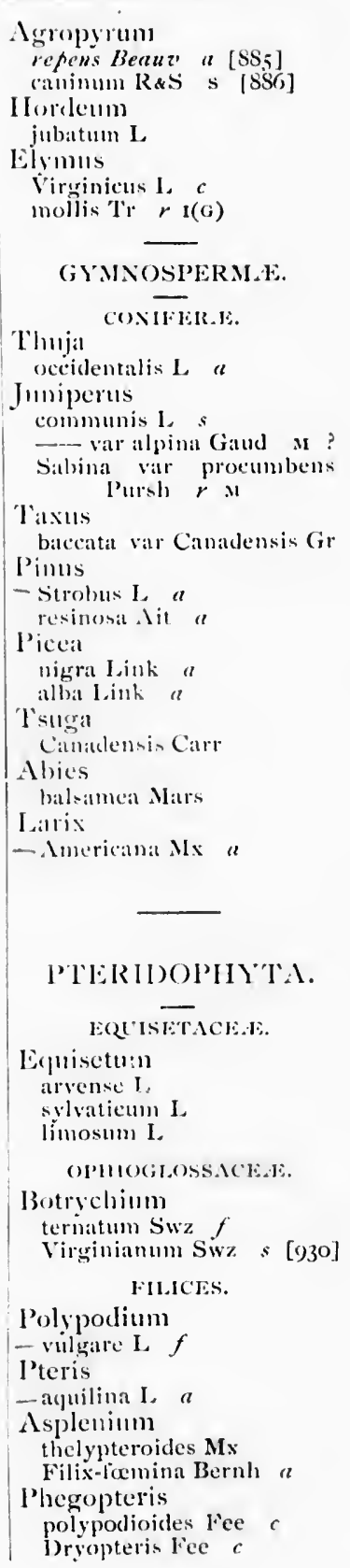 \\
\hline
\end{tabular}




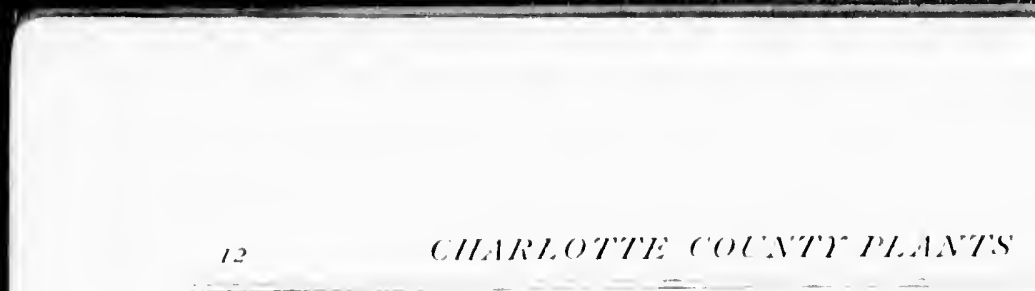

Ispridium

acrostichoides Sive

Noveloracense Swy

(Thelyperi-sw\% so;)

cristitum Sw\%

-ppinulo-mu var it III) li:t(t) o

Cinfepteris

irateilis Bernh ,

lublififera Bermh * ()mocleat

sm-ibili, L. $c$

Struthiopteri- Ilotlim [020]

IVorrelsial

Hvensin RBr f

i- Dicksomial

- pilominscula Willa a lyzl

()-mumbla

recilio $\mathrm{L}$

Clatytoniama I. c

- cimnimonnea 1 ,

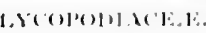

I. yeopodium

Incidulum Ms ,

antutimแtul.

dendroidemin Mx "

clavalumi 1, r

complanatum L.

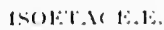

isseles

echincepora val Bratunii lingl J(1),

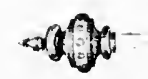





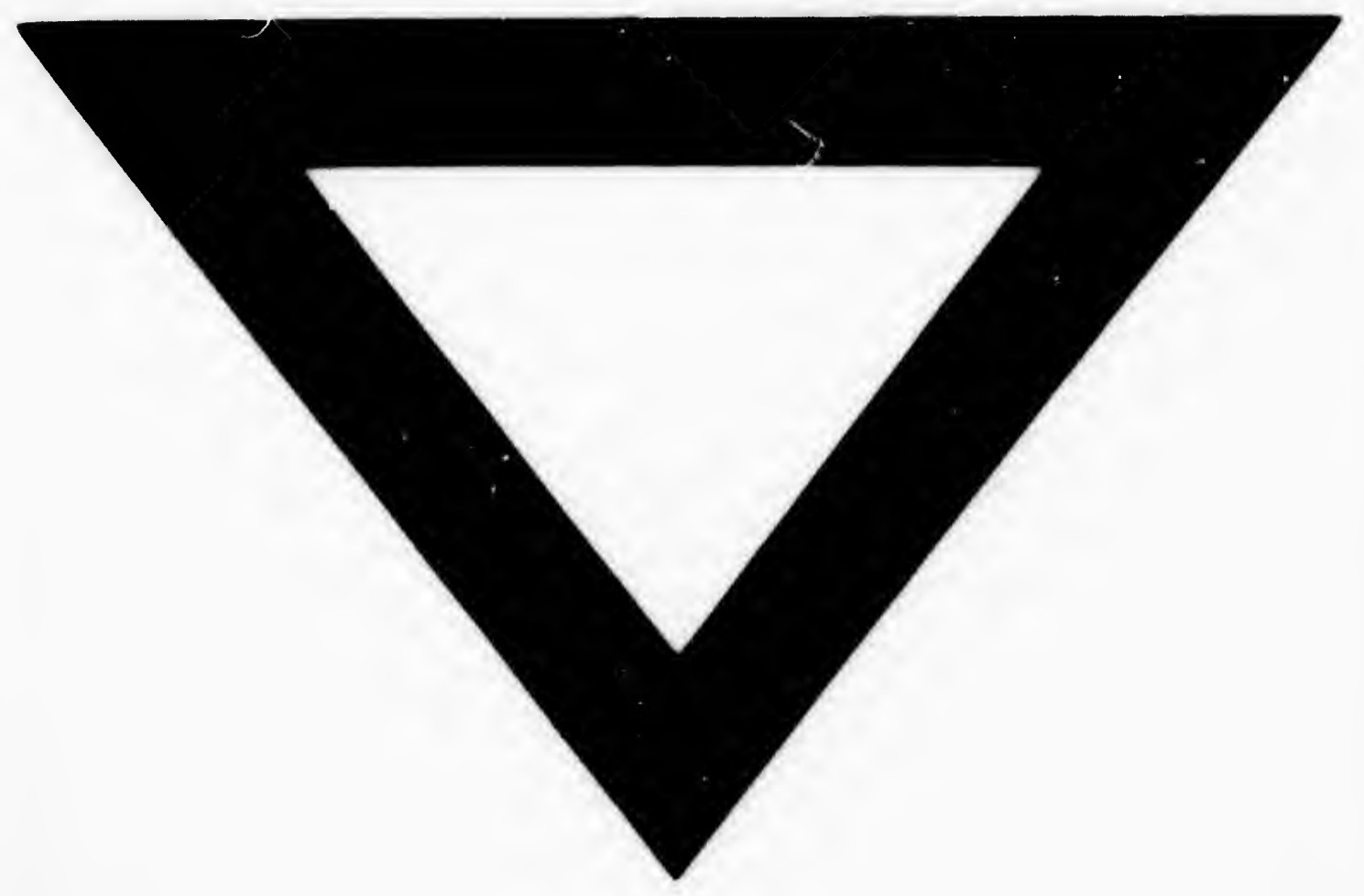

